\title{
Medical Image Segmentation using Modified Morphological Reconstruction
}

\author{
A.Nithya \\ Research Scholar,Annamalai University \\ Chidambaram, India
}

\author{
R.Kayalvizhi \\ Professor, Annamalai University \\ Chidambaram, India
}

\begin{abstract}
The objective of this research is to improve the accuracy of object segmentation in medical images by constructing an object segmentation algorithm. Image segmentation is a crucial step in the field of image processing and pattern recognition. Segmentation allows the identification of structures in an image which can be utilized for further processing. Both region-based and object-based segmentation are utilized in a robust and principled manner. Gradient based MultiScalE Graylevel mOrphological recoNstructions (GSEGON) is used for segmenting an image. SEGON roughly identifies the background and object regions in the image. The proposed method takes advantage of segmentation of both gray scale image and color image.
\end{abstract}

Keywords: G-SEGON, Grey level mesh, opening and closing reconstruction, gradient, K-mean clustering, accuracy.

\section{INTRODUCTION}

Image segmentation is a very commonly used and important step in image analysis and computer vision [11]. In the classical computer vision paradigm, the problems of image segmentation and object behaviour classification lie at different levels of abstraction. The purpose of image segmentation is to decompose an image domain into a number of disjoint regions so that the features within each region have visual similarity, strong statistical correlation and reasonably good homogeneity. At a basic level, segmentation aims at extracting meaningful objects from the target image(s). It allows the identification of structures in an image which can be utilized for further processing. Although numerous methods are available for image segmentation, it is still under problem because of its complexity and inadaptability.

Usually, image segmentation algorithms are classified into two types, supervised and unsupervised. Unsupervised algorithms are fully automatic and partition the regions in feature space with high density [8]. The different unsupervised algorithms are Feature-Space based Techniques, Clustering (K-means algorithm, C-means algorithm, E-means algorithm), Histogram thresholding, Image-Domain or Region Based Techniques (Split-and-merge techniques, Region growing techniques [9], Neural-network based techniques, Edge Detection Technique), Fuzzy Techniques [10], Hybrid techniques, etc. In recent years, mathematical morphology is a well-known technique used in image processing and computer vision [11] [12] [13]. Set theoretic, shape oriented approach treats the image as a set and the kernel of operation, commonly known as structuring element (SE), as another set. Different standard morphological operations namely dilation, erosion, opening, closing etc. are basically set-theoretic operations between these two sets. On the other hand, most spatial domain image processing techniques use the notion of local neighbourhood which does not take care of the scale of the object contained in that neighbourhood. The objects in an image should be processed as per their scales. Thus the need for processing the image based on the size or scale has initiated several multiscale and multi resolution techniques.

Multiscale and multiresolution techniques extract scale specific information from the image and integrate them to produce desired output. The entire process may be linear or nonlinear and accordingly it gives rise to a linear or nonlinear scale space representation of the image [14]. Image and video segmentation is considered as an essential issue in the image coding filed. A large number of previous methods try to solve the segmentation problem from a certain perspective, e.g., threshold, template matching, region growing, edge detection and clustering. These methods have been proven to be successful in many applications, but none of them are generally applicable to all images and moving objects and different algorithms are usually not equally suitable for a particular application.

\section{PROPOSED METHOD}

In this section, the proposed method to segment the object from the image is described. To perform this operation GSEGON is used which provides segmentation more accurately than the existing method. First the background region is roughly detected by using opening and closing reconstruction, then the mesh was constructed over the region. After constructing mesh the image is converted into gradient and $\mathrm{K}$ mean clustering algorithm is used to segment object by removing the background (BG). After segmentation both gradient segmented image and gray scale segmented image are compared. Then the combined result is refined to get object. The overall block diagram of the proposed technique is shown in Fig.1

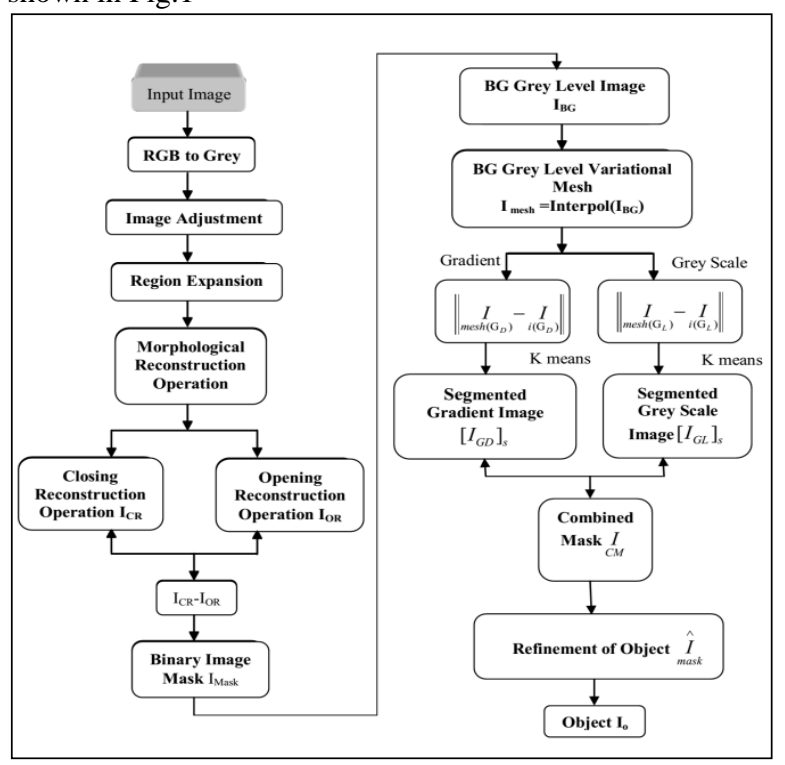

Fig1.Overall Block Diagram Of Proposed Technique 
The main contributions of the proposed technique are

1) The object can be segmented from both images and videos.

2) Object segmentation is done through k-means clustering for gradient and grey level images.

3) Both gradient and grey level segmentation is performed to improve the segmentation accuracy.

4) Then the gradient and grey level segmented image is combined and the object is refined.

\subsection{Pre-processing phase}

Pre-processing is necessary to remove the noises present in the image and to get accurate segmentation of object. The image cannot be applied directly for performing proposed technique due to the presence of noise in the image. To make it suitable for further operation pre-processing is done. It involves several processes such as RGB to grey level conversion, Image adjustment, and Region expansion.

- $\quad R G B$ to grey level conversion: In this step of preprocessing the input image or frame $I_{i}$ is first converted into Grey level image . The image consists of grey shades, based upon the intensities the variation takes place from black to white. For the conversion of RGB to grey level representation, first step is to obtain the red, green and blue values. Then add the percentage of red, green and blue values.

- Image adjustment: In this step, quality of the image is improved by using image adjustment. Image adjustment is used for image enhancement.

- After image adjustment, region expansion is performed to get accurate position of object in the image. The result obtained in this process is taken for further process.

\subsubsection{Morphological reconstruction operation}

After pre-processing, multi-scale morphological operations is used to extract the features in the image $I$. Dual multi-scale

reconstruction operation was performed to segment object in the image. The image is first segmented by opening the image using the structural element of particular size. Then the opening reconstruction operation is performed until the original shape is recovered. To extract different object in the image particular structural element is used. This operation involves both opening and closing operation.

\subsubsection{Opening:}

For opening operation consider a binary image $\underset{b i n}{I}$ with structural element $f$ in which the opening operation is performed by taking the erosion of the image $I_{\text {bin }}$ then the result obtained undergoes dilation with structural element $f$, which is given as:

$$
\underset{\text { bin }}{I \circ f}=(\underset{b i n}{I} \Theta f) \oplus f
$$

Where, $\underset{\text { bin }}{I}$ is the binary image.

$f$ is the structural element,
Closing:

For closing operation consider a binary image $\underset{b i n}{I}$ with structural element $f$ in which the opening operation is performed by taking the dilation of the image $I_{\text {bin }}$ then the result obtained undergoes erosion with structural element $f$, which is given as:

$$
\underset{\text { bin }}{I \bullet f}=(\underset{b i n}{I} \oplus f) \Theta f
$$

Where, $\underset{b i n}{I}$ is the binary image.

$f \quad$ is the structural element

\subsubsection{Back ground grey level variation}

The technique that used in the object identification is extended to segment the object and their grey level by use both open and close reconstruction. To get stable outcome the reconstructed $\mathrm{OR}(\mathrm{CR})$ operation is not iterated completely so that the convex (concave) grey level variation of the image can be located. The object boundaries can be detected by using proper structural element. Multi-scale open and close reconstruction was performed to obtain proper structural elements to locate the concave and convex grey level variation. The grey level variation of the processed image varies from the grey level of the input image for this detaching process is carried out. To obtain the stable grey-level variation morphological opening and closing operation is done simultaneously. This finds whether the concave and convex grey level variation is located or not. While performing OR (CR) operation with different structural element in the image false segmentation may occurs to avoid this smooth structural element operation is done across the boundary. Background grey level variation is identified by subtracting the image obtained from close operation with the open reconstruction operation.

$$
\underset{B G}{I}=\underset{C R}{I}(i, j)-\underset{O R}{I}(i, j)
$$

Where ${ }_{B G}^{I}$ - Background variation of image.

$I_{C R}^{I}, \underset{O R}{I}$ - Image obtained by open and close operation.

$(i, j)$ - is the set of pixels.

\subsection{Object region segmentation phase}

Based on the OR (CR) operation the object in the image can be segmented by following three steps.

\subsubsection{Initialization of object region}

Binary image mask $\underset{\text { mask }}{I}$ is the initial process of image segmentation. Binary Mask partitions the grey level image into object and background regions using top hat or bottom hat operation. It provides an outline of the object in the image. 


\subsubsection{BG grey level variational mesh}

In this step, a background grey level mesh was constructed across the boundary region between the object and background in the image. When the background and the object regions are closely present in the image then the structural element cannot differentiate the object and back ground, so to overcome this problem background variational mesh was constructed across the boundary. The grey level variational mesh was constructed by making use of isolated data points in the image. To construct grey level variational mesh, Lagrangian interpolant algorithm is used.

$$
\underset{\text { mesh }}{I}=\operatorname{int} \operatorname{erp}\left(I_{B G}\right)
$$

After constructed mesh, image segmentation process is done through k-means clustering to segment the object for gradient and grey scale images.

\subsubsection{Gradient image segmentation using $k$-means clustering}

In this step both gradient and k-mean operation to segment object from the image are used. Here, gradient operation is performed on $\underset{m e s h}{I}$ and original image $I_{i}$ to extract visual information. A gradient magnitude operator detects the amplitude edges at which pixel change their gray levels suddenly. Generally, image gradient is directional change in the intensity or colour in an image.

Gradient image ${ }_{G D}^{I}$ is obtained by taking the gradient of the input image and then subtracting it with gradient of the BG grey level variational mesh.

Where,

$$
\underset{G D}{I}=\|\underset{m e s h(G D)}{I}-\underset{i(G D)}{I}\|
$$

$\underset{G D}{I}$ is the result gradient image

$\underset{m e s h(G D)}{I}$ is the gradient of the BG grey level variational mesh

$\underset{i(G D)}{I}$ is the gradient of the original image

After calculating $I_{G D}$, the object region segmentation process is performed using k-means clustering. The clustering can be improved by assuming that neighbouring pixels have a high probability of falling into the same cluster. In image segmentation application, the observations are based on the pixels in the image plane. Consequently, K-mean clustering is applied for $I_{G D}$ and then segmented $\left[I_{G D}\right]_{S}$ is obtained.

The K-means segmentation algorithm is follows:

Step 1: Place $\mathrm{K}$ points into the space represented by the objects that are being clustered. These points represent initial group centroids.

Step 2: Assign each object to the group that has the closest centroid by following objective function

$$
J=\sum_{j=1}^{k} \sum_{i=}^{n}\left\|x_{i}^{(j)}-v_{j}\right\|^{2}
$$

Where,

$\left\|x_{i}^{(j)}-v_{j}\right\|^{2}$ is a chosen distance measure between a data point $x_{i}$ and the cluster centre $v_{j}$

Step 3: When all objects have been assigned, recalculate the positions of the K centroids.

Step 4: Repeat Steps 2 and 3 until the centroids no longer move. This produces a separation of the objects into groups from which the metric to be minimized can be calculated.

\subsubsection{Gray level image segmentation using $k$ means clustering}

The resultant grey scale image $I_{G L}$ is obtained by subtracting the input grey scale image with the background (BG) grey level mesh.

$$
I_{G L}=\|\underset{\operatorname{mesh}(G L)}{I}-\underset{i(G L)}{I}\|
$$

Where, $I_{G L}$ is the result grey level image

$$
\underset{I}{I} \text { is the grey level image of the BG grey level }
$$
variational mesh

$$
\underset{i(G L)}{I} \text { is the original grey level image }
$$

Consequently, K-mean clustering is applied for $I_{G L}$ and then segmented $\left[I_{G L}\right]_{S}$ is obtained from the $I_{G L}$.

\subsection{Majority selection and Refinement phase}

After object region segmentation $\left[I_{G D}\right]_{S}$ and $\left[I_{G L}\right]_{S}$, the combined mask $I_{C M}$ is done by majority selection process. In this step the segmented image obtained from both the grey level and the gradient operation are combined to get accurate segmentation of object in the image. For this we use majority selection procedure which is carried out by following steps first particular group of pixels in both images is taken to consideration then compare the pixel group in both grey level segmented image and the gradient segmented image. If both the pixel has same value i.e. 0 or 1 then no change is needed. For different value, the majority value in the neighbourhood pixels of particular pixel is taken and the pixel value is replaced by the majority value.re. This procedure is repeated until the object is fully recovered from the image.

1. Start

2. Compare both segmented gradient image $\left[I_{G D}\right]_{S}$ and Grey scale image $\left[I_{G L}\right]_{S}$

3. Consider every pixel $\mathrm{H}_{C}$ in both images

4. If $\left[I_{G D}\left(H_{c}\right)\right]_{s}-\left[I_{G L}\left(H_{c}\right)\right]_{s}$

5. No change

6. Else

7. Replace the pixel with majority of the neighbourhood pixel.

8. end 


\subsubsection{Refinement of object and boundary region}

After performing the majority selection the object and boundary region in the image was refined. For boundary refinement the coherent grey level between the input image and the back ground grey level mesh is taken as back ground pixel and for region refinement the grey level between the regions is taken. To avoid poor matching between the obtained image and the original image, coherent region justification procedure was done and the final object was extracted from the image $I$.

$$
I=\underset{\text { mask }}{I} \otimes I_{i}
$$

Where

$$
\begin{aligned}
& \hat{I}=\operatorname{refine}[\underset{i}{I}, \underset{C M}{I}], \\
& \underset{\text { mask }}{\hat{I}}=\operatorname{sign}\left(\frac{\left\|\underset{C M}{I}-I_{i}\right\|}{\left|I_{i}\right|}-\underset{T}{D}\right),
\end{aligned}
$$

\section{RESULTS \& DISCUSSIONS}

The results obtained from the proposed G-SEGON technique is discussed in this section.

\subsection{Dataset description}

The proposed technique was developed using mat lab version (7.12). This technique is performed in a windows machine having configuration Intel ${ }^{\circledR}$ core i5 processor, $3.20 \mathrm{GHz}, 4$ GB RAM, and the operating system is Microsoft Window7 professional.

Dataset description: For this proposed technique 6 images are cosidered. Each image is about $512 \times 512$ pixel resolution. Test images are publically available. The considered 6 input images for proposed technique are given in Figure.2.
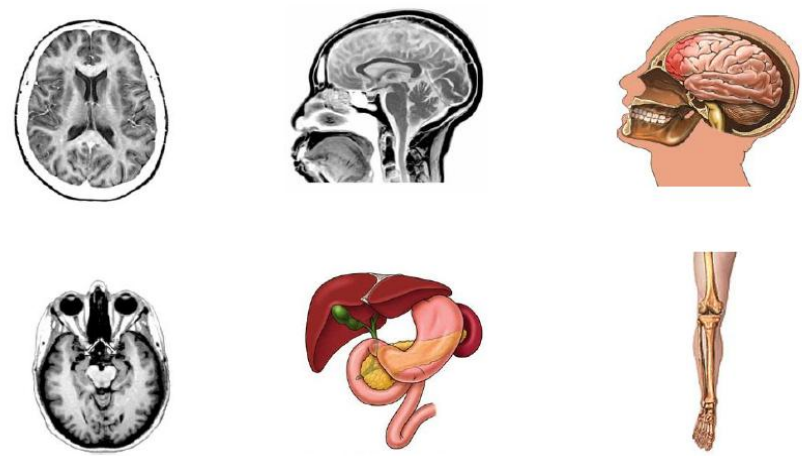

Figure.2 Input Images For Proposed Technique

\subsection{Evaluation metrics}

In this the accuracy of the image segmentation is calculation by taking the ratio of manually segmented image region to the proposed image region. The formulae to calculate accuracy is given as

$$
\text { Accuracy }=\frac{(A \cap B)}{(A U B)}
$$

Where $A$ is the manually segmented region

$B$ is the proposed image region

\subsection{Simulation results}

The Simulated output in Table.1 shows the segmentation of ten images by existing method and proposed method which is given below references

\subsection{Comparative analysis}

In this paper, proposed G-SEGON technique was compared against region growing algorithm (existing). The accuracy of proposed method is high compared to existing method. The comparative analysis of the 10 images is given in the following figures $4(\mathrm{a})$ to $4(\mathrm{j})$. The plot in the figure includes the threshold and the segmentation accuracy. The graph is drawn by varying the threshold value in open and close reconstruction operation. The corresponding accuracy is calculated for different threshold value. From the figure 4( a) to 4( i) the accuracy of both existing and proposed method is individually compared for ten images. The figure $4(\mathrm{k})$ gives the average accuracy of ten images in which the threshold value is varied from 1 to 5 . From the figure it is clear that in existing method, for small threshold value the accuracy is very low, when compared to the G-SEGON. In G-SEGON the accuracy remain high for all the threshold value from 1 to 5 . Comparative analysis of images is given below table1.

\section{CONCLUSION}

In this paper an object segmented algorithm G-SEGON is developed to improve the accuracy of object segmentation. G-SEGON combined with K-means clustering segmentation provides an efficient method to refine object boundaries. To find the efficiency of the proposed technique the accuracy is calculated. From the comparison of ten images it is clear that the accuracy of existing method is far behind the proposed method. The future work is to extend G-SEGON for medical images.

\section{REFERENCES}

[1] D. Comanicu and P. Meer,"Mean shift: A robust approach toward feature space analysis", Pearson Education Ltd., IEEE Trans. Pattern Anal. Mach. Intell., vol. 24, no. 5, pp.603-619, May 2002.

[2] Y. Deng and B. S. Manjunath,'Unsupervised segmentation of colour texture regions in images and video," IEEE Trans. Pattern Anal. Mach. Intell., vol. 23, no. 8, pp. 800810, Aug. 2001.

[3] M. P. Pathegama and. Gl,"Edge-end pixel extraction for edge based image segmentation", in Proc. World Acad. Sci. Eng. Technology., vol. 2, pp. 164-167, Jan.2005.

[4] D.Sasirekha and Dr.E.Chandra, "ContourEnhanced Techniques for PDF Image Segmentation and Text Extraction,” International Journal of Computer Science and Information Security, vol.10, no.9, pp.7-27, 2001

[5] Velthuizen.R et al.,'Unsupervised measurement of brain tumour volume on MR images," Journal of magnetic resonance imaging, vol.4, pp. 594-605, 1995.

[6] Vinitski.S et al.: Improved intracranial lesion characterization by tissue segmentation based on a 3D feature map, Journal of magnetic resonance in medicine, vol.5, pp.457-469, 1997. 
[7]Gerig. G.: Automated segmentation of dual-echo MR head data, Proceedings of IPMI, pp. 175-185, 1991.

[8] M.C.Jobin Christ and Dr.R.M.S.Parvathi, "Magnetic resonance Brain image segmentation," International Journal of VLSI design \& Communication Systems, vol.3, no.4, pp.121-133, 2012.

[9] P. Salembier and F. Marques, "Region-based representations of image and video-segmentation tools for multimedia services, "IEEE Trans. Circuits Syst. Video Technol., vol. 9, no. 8, pp. 1147-1169, Dec. 1999.

[10] Fitsum Admasua, Stephan Al-Zubia, Klaus Toenniesa, Nils Bodammerb and Hermann Hinrichsb, "Segmentation of Multiple Sclerosis Lesions from MR Brain Images Using the Principles of FuzzyConnectedness and Artificial Neuron Networks", In Proceedings of International Conference on Image Processing, Barcelona, Spain, Vol. 3, 2003.
[11] Jiann-Jone Chen, Chun-Rong Su, W. Eric L. Grimson, Jun-Lin Liu, and De-Hui Shiue,"Object Segmentation of Database Images by Dual Multiscale Morphological Reconstructions and Retrieval Applications",IEEE Transactions On Image Processing, Vol. 21, No. 2, February 2012.

[12] P. T. Jackway,"Morphological scale space, in Proc. 11th IAPR Int. Conf. Pattern Recognition, The Hague, The Netherlands, September 1992.

[13] P. Salembier and M. Pardas,'Hierarchical morphological segmentation for image sequence coding," IEEE Trans. Image Processing, vol. 3, pp.639-651, Sept. 1994.

[14] P.Mirunalini, R.Nanmozhi,"Segmentation Using Multiscale Morphological Reconstruction,"SSN College of Engg, Chennai, 2013.

Table 1: Proposed G-SEGON segmentation results against existing method for input images

Input images Existing method




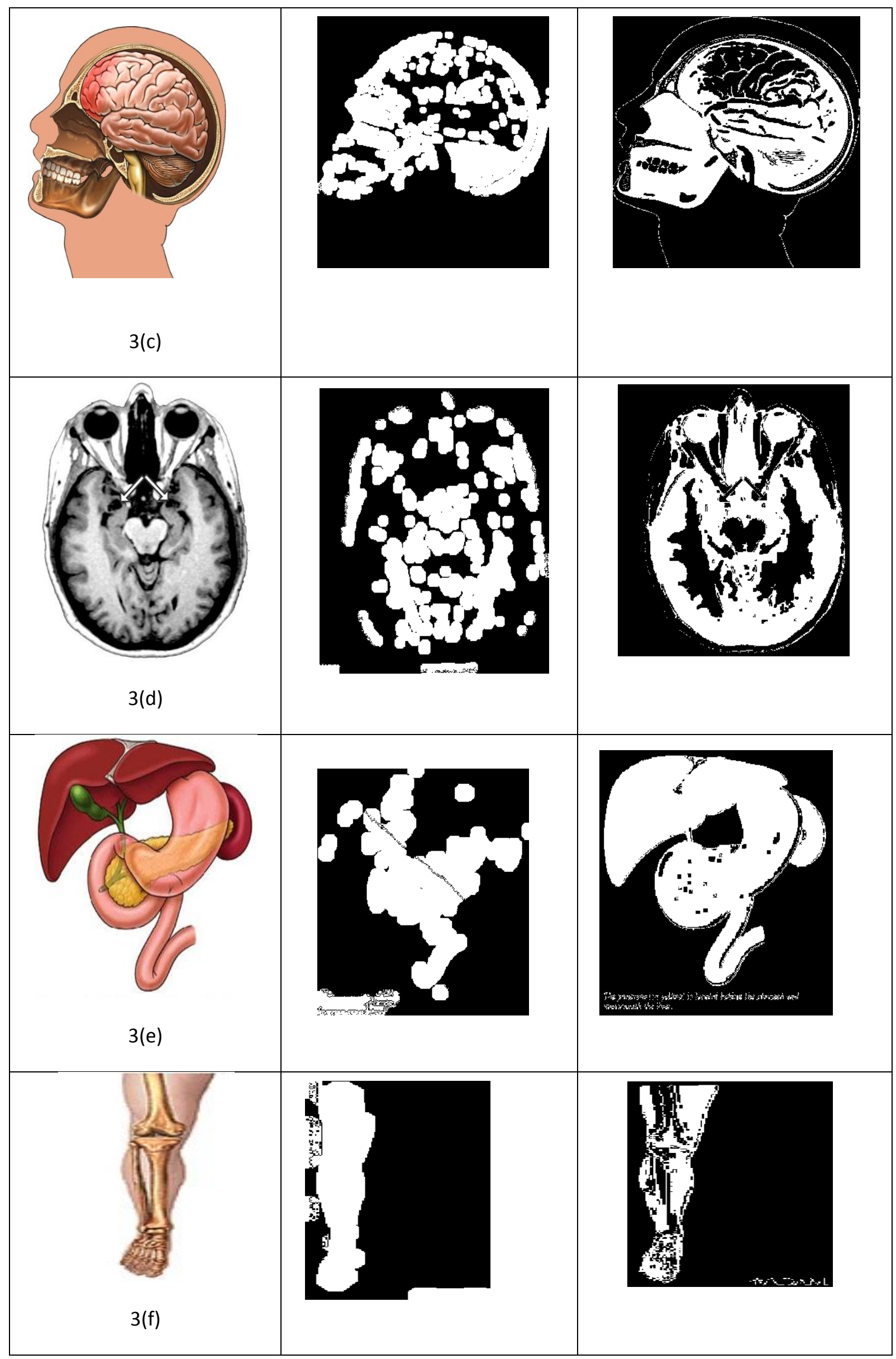




\section{Comparative analysis of medical images}

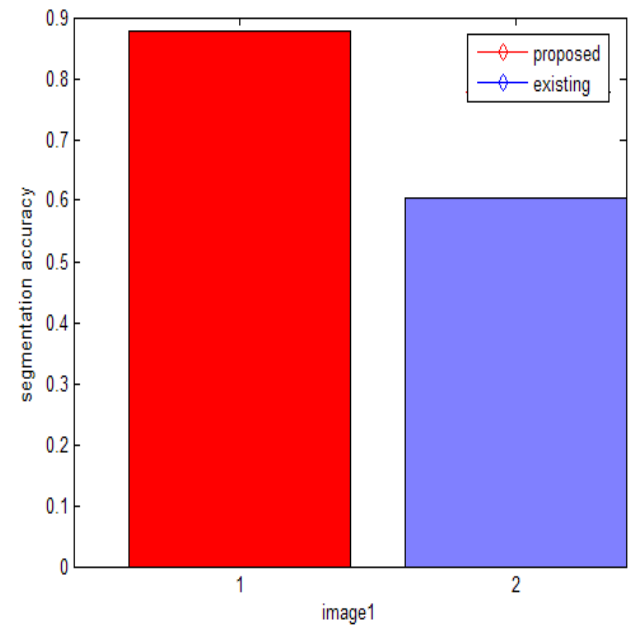

Fig 4a) Comparison of image 3(a)

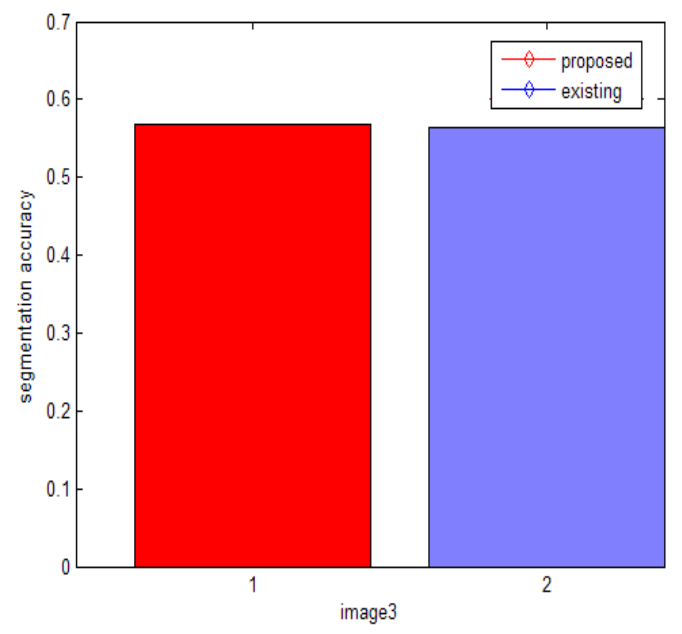

Fig 4c) Comparison of image 3(c)

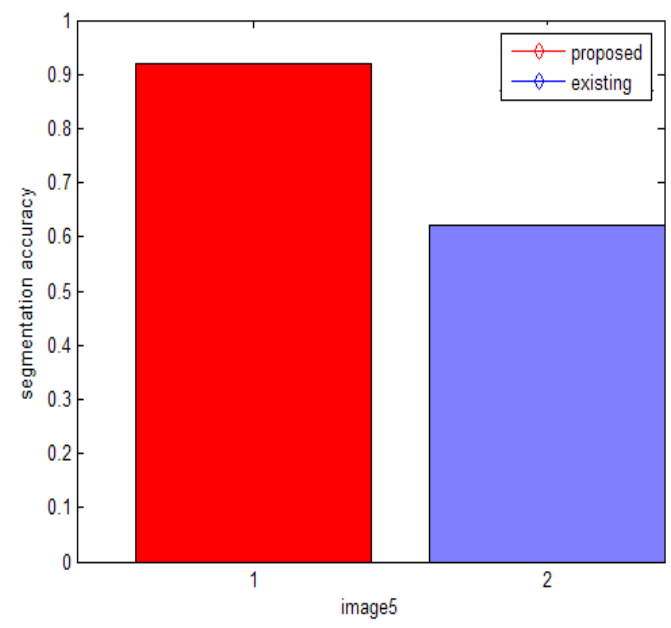

Fig 4e) Comparison of image 3(e)

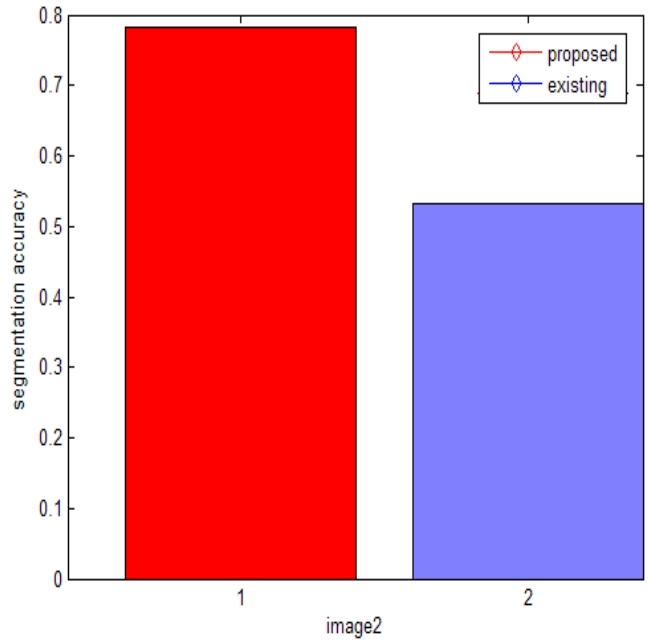

Fig 4b) Comparison of image 3(b)

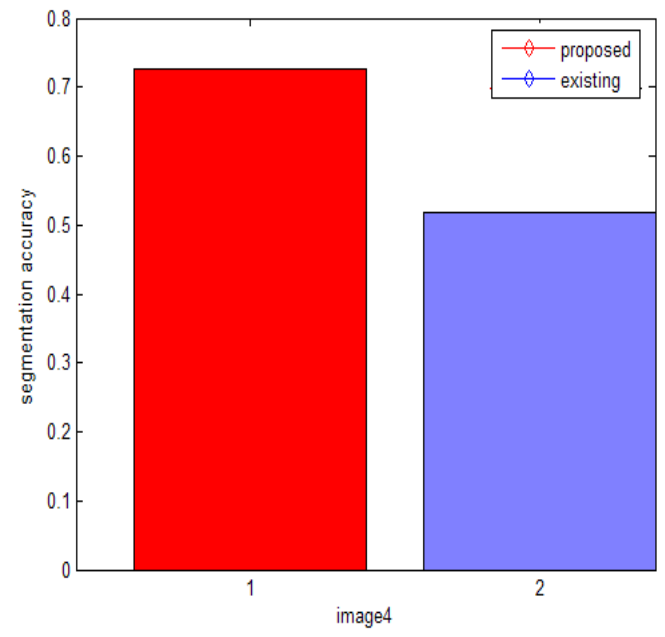

Fig 4d) Comparison of image 3(d)

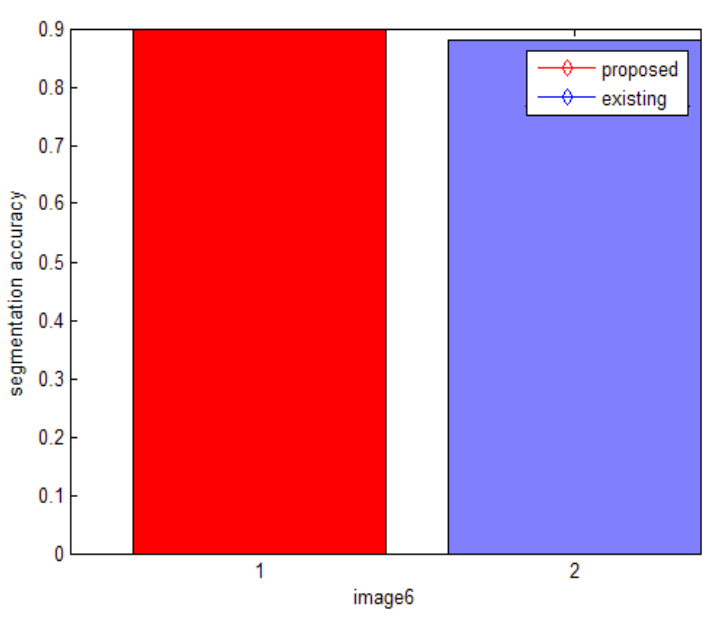

Fig 4f) Comparison of image 3(f) 